\title{
Phase discrimination in CdSe structures by means of Raman scattering
}

\author{
Ramon Cuscó $^{*}, 1$, V. Consonni ${ }^{2}$, E. Bellet-Amalric ${ }^{3}$, R. André ${ }^{4}$, L. Artús ${ }^{1}$ \\ ${ }^{1}$ Institut Jaume Almera (ICTJA-CSIC), Consejo Superior de Investigaciones Científicas, Lluís Solé i Sabarís s.n., 08028 Barcelona, \\ Spain \\ 2 Université Grenoble Alpes, CNRS, LMGP, F-38016 Grenoble, France \\ ${ }^{3}$ Université Grenoble Alpes, F-38000 Grenoble, France and CEA, INAC-PHEILQS, Nanophysique et Semiconducteurs group, F-38000 \\ Grenoble, France \\ ${ }^{4}$ Université Grenoble Alpes, F-38000 Grenoble, France and CNRS, Institut Néel, Nanophysique et Semiconducteurs group, F-38000 \\ Grenoble, France
}

Received XXXX, revised XXXX, accepted XXXX

Published online XXXX

Key words: CdSe structures, wurtzite phase, zincblende phase, Raman scattering

* Corresponding author: e-mail lartus@ija.csic.es, Phone: +34-93-4095410, Fax: +34-93-4110012

Raman spectra of epitaxial layers of CdSe grown by molecular beam epitaxy have been measured for the cubic (zincblende) and hexagonal (wurtzite) phases. The Raman spectra are examined in the light of density functional calculations for these two highly similar structures. Characteristic Raman frequencies and spectral features associated with the different symmetry are discussed and reliable criteria for phase discrimination based on Raman spectroscopy are proposed. Although
LO frequencies are virtually identical in both structures and may be affected by size effects, the observation of a low energy $E_{2}$ mode at $33 \mathrm{~cm}^{-1}$ unambiguously identifies the wurtzite structure and can be used as a specific fingerprint to distinguish between these two phases in CdSe-based nanostructures. The slightly lower LO frequency measured in the zincblende epitaxial layer is ascribed to residual tensile strain.

Copyright line will be provided by the publisher

1 Introduction CdSe is a semiconductor material $\left(E_{g} \sim 1.7 \mathrm{eV}\right)$ which has a key role in the development of high efficiency solar cell nanostructures. As CdSe displays a high absorption in the solar spectrum range which is complementary to that of $\mathrm{ZnO}$, it is widely used for the sensitization of $\mathrm{ZnO}$ nanowires to solar light [1]. Band gap tuning can be achieved by quantum confinement in nanostructures with different sizes and shapes, and since the $\mathrm{ZnO} / \mathrm{CdSe}$ heterostructure yields a type II band alignment, the absorption profile of $\mathrm{ZnO} / \mathrm{CdSe}$ core-shell nanowires covers wavelengths longer than those corresponding to the band gap of the CdSe shell and display an increased carrier collection efficiency.

CdSe crystallizes in two lattice structures: the cubic zincblende (ZB) structure and the hexagonal wurtzite (W) structure. In bulk, ZB is the stable low-temperature phase and transforms to $\mathrm{W}$ phase above a critical temperature of $95{ }^{\circ} \mathrm{C}[2]$, but depending on the growth conditions samples can be obtained at ambient pressure in either ZB or W forms. Although single-crystal bulk CdSe grown by molecular beam epitaxy (MBE) usually adopts the $\mathrm{W}$ form, $\mathrm{CdSe}$ single-crystal epilayers with ZB structure can be obtained by MBE growth on ZB substrates [3]. By contrast, small CdSe nanocrystals tend to exhibit the ZB phase. Electrodeposited CdSe shells on $\mathrm{ZnO}$ nanowires also revealed $\mathrm{ZB}$ structure for as-deposited samples and samples annealed at temperatures below $350{ }^{\circ} \mathrm{C}$ [4]. However, annealing of the $\mathrm{ZnO} / \mathrm{CdSe}$ core-shell structures at temperatures above $350{ }^{\circ} \mathrm{C}$ results in the formation of the $\mathrm{W}$ phase, which becomes the dominant phase at $400{ }^{\circ} \mathrm{C}$ [4]. 
Crystal structures of polytypes that share a common crystal facet can be used to achieve branching, as in the case of tetrapods consisting of a ZB core and four projecting $\mathrm{W}$ nanorods [5]. Seeded growth of CdSe/CdS nanostructures allows the shape of nanocrystal heterostructures to be tailored into nanorods or tetrapods by using $\mathrm{CdSe}$ nanocrystals with $\mathrm{W}$ and $\mathrm{ZB}$ structure, respectively, as seeds. Typical AM1.5 solar power conversions of $\sim 2.1 \%$ are obtained in CdSe tetrapod/polymer hybrid solar cells [6]. Recently, CdSe/CdS core-shell tetrapods have been shown to exhibit multicolor emission associated with a higher energy transition that is allowed via spatially indirect recombination [7]. This opens exciting possibilities for controlling carrier localization at the nanoscale in photovoltaic and solar fuel applications.

The characterization of CdSe nanocrystals is primarily done by X-ray diffraction (XRD). Since the ZB and WZ CdSe phases possess very similar XRD patterns, any strain and/or nano-sized effects make their distinction not straightforward, especially when local epitaxy is involved, which requires the use of sophisticated XRD measurements using specific geometries. Optical measurements are also employed to characterize $\mathrm{ZB}$ and $\mathrm{W}$ phases. The gap between the first and second exciton transitions is wider for $\mathrm{ZB}$ than $\mathrm{W}$ CdSe nanocrystals, and the peak positions for the exciton transitions with higher energies is also different [8]. However, differences in shape and position of the absorption edge can be influenced by size quantization effects and structural disorder [9].

Recently, Kelley et al. [10] made an attempt to differentiate between both CdSe phases by performing a resonant Raman study on spherical nanospheres. However, they could not find any significant difference neither in the frequencies nor in the resonant intensities of the respective LO peaks. In this letter, we have addressed the distinction between the $\mathrm{W}$ and $\mathrm{ZB}$ phases of CdSe by performing Raman scattering studies on MBE grown CdSe films. This allows us to establish sound criteria to differentiate both phases since it avoids possible effects associated with nanosize crystallites such as surface reconstruction, confinement effects, and/or lattice contraction [11], and the scattering geometry implies a well defined phonon wavevector that rules out possible quasimode effects usually present in nanostructures [12].

2 Experiment The $\mathrm{W}$ and $\mathrm{ZB}$ CdSe epitaxial layers were grown on GaAs (111)B and $\mathrm{ZnTe}(100)$ substrates, respectively, by molecular beam epitaxy (MBE) using a Riber ultra-high vacuum chamber. A GaAs buffer layer was grown at $630{ }^{\circ} \mathrm{C}$ under As-rich conditions. Subsequently, the (0001) W CdSe epilayer was deposited at $260{ }^{\circ} \mathrm{C}$ under Se-rich conditions. Prior to the growth of the ZB CdSe epilayer, a ZnTe buffer layer was grown at $380{ }^{\circ} \mathrm{C}$ under $\mathrm{Zn}$-rich conditions. Subsequently, a $550 \mathrm{~nm}$ thick (100) ZB CdSe epilayer was deposited at $280^{\circ} \mathrm{C}$ under Se-rich conditions.
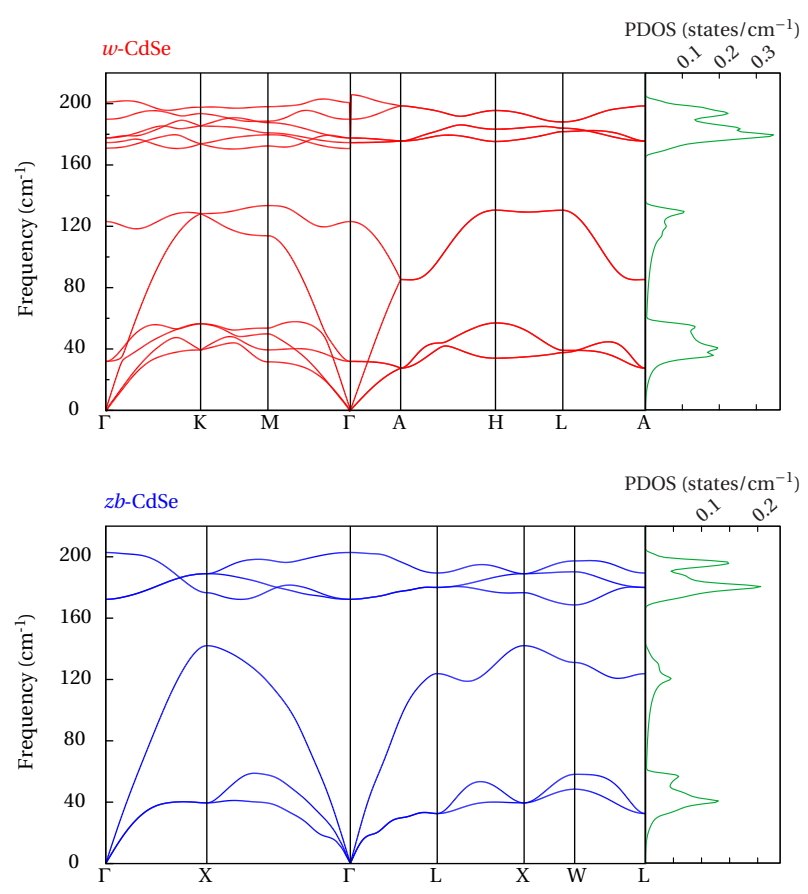

Figure 1 Phonon dispersion relations and phonon density of states for wurtzite (top panel) and zincblende (lower panel) CdSe.

The samples were characterized by XRD using a RIGAKU Smartlab diffractometer equipped with a fivecircle goniometer. For the $\mathrm{W}$ sample, the lattice parameter values $a=4.299 \AA$ and $c=7.0127 \AA$ were determined, leading to a $c / a$ ratio of 1.631 , in close agreement with generally accepted values for bulk W-CdSe [13], indicating that the studied epilayer is mainly relaxed. For the $\mathrm{ZB}$ epilayer grown on the $\mathrm{ZnTe}(100)$ substrate, an in-plane lattice constant $a_{\perp}=6.102 \AA$ was determined, whereas the out-of-plane lattice constant was found to be $a_{\|}=6.055 \AA$, suggesting the presence of a residual tensile biaxial strain. The Raman spectra were recorded in unpolarized backscattering configuration using a Jobin-Yvon T64000 spectrometer using the $514.5 \mathrm{~nm}$ line of an $\mathrm{Ar}^{+}$ laser as excitation.

\section{Results}

3.1 DFT calculations To aid in the interpretation of the Raman spectra, density functional theory (DFT) calculations of the phonon dispersion were carried out for both structures. The calculations were performed in the local density approximation (LDA) using the ABINIT code [14]. A $6 \times 6 \times 4$ Monkhorst-Pack grid was used for integration over the Brillouin zone of the $\mathrm{W}$ structure, with an energy cutoff of 60 hartree. A full structural relaxation of the W CdSe lattice yields $a=4.290 \AA$ and $c / a=1.632$. These values are in good agreement with the lattice parameters measured by XRD in our sample. For the ZB structure, we used a $(6 \times 6 \times 6) \mathbf{k}$-point grid with an energy cutoff of 80 hartree. A lattice parameter $a=6.065 \AA$ was obtained for 
Table 1 Experimental and calculated phonon frequencies in units of $\mathrm{cm}^{-1}$ of the Raman active modes of W and ZB CdSe. Calculated values were corrected for the LDA overestimation of the dielectric constant.

\begin{tabular}{|c|c|c|c|c|c|c|c|c|c|c|c|c|}
\hline \multirow[t]{2}{*}{ ZB CdSe } & & & & & \multicolumn{4}{|c|}{ 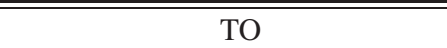 } & \multicolumn{4}{|c|}{$\overline{\mathrm{LO}}$} \\
\hline & & & & & \multicolumn{2}{|c|}{$\begin{array}{c}\text { Exp. } \\
-\end{array}$} & \multicolumn{2}{|c|}{$\begin{array}{l}\text { Calc. } \\
172.3\end{array}$} & \multicolumn{2}{|c|}{$\begin{array}{c}\text { Exp. } \\
205.2\end{array}$} & \multicolumn{2}{|c|}{$\begin{array}{l}\text { Calc. } \\
211.9\end{array}$} \\
\hline \multirow[t]{3}{*}{ W CdSe } & \multicolumn{4}{|c|}{$E_{2}$} & \multicolumn{4}{|c|}{ TO } & \multicolumn{4}{|c|}{ LO } \\
\hline & \multicolumn{2}{|c|}{ low } & \multicolumn{2}{|c|}{ high } & \multicolumn{2}{|c|}{$A_{1}$} & \multicolumn{2}{|c|}{$E_{1}$} & \multicolumn{2}{|c|}{$E_{1}$} & \multicolumn{2}{|c|}{$A_{1}$} \\
\hline & $\begin{array}{l}\text { Exp. } \\
33.0\end{array}$ & $\begin{array}{l}\text { Cal. } \\
31.9\end{array}$ & $\begin{array}{c}\text { Exp. } \\
174.0\end{array}$ & $\begin{array}{l}\text { Calc. } \\
177.6\end{array}$ & $\begin{array}{c}\text { Exp. } \\
-\end{array}$ & $\begin{array}{l}\text { Calc. } \\
170.7\end{array}$ & $\begin{array}{c}\text { Exp. } \\
-\end{array}$ & $\begin{array}{c}\text { Cal. } \\
174.6\end{array}$ & $\begin{array}{c}\text { Exp. } \\
-\end{array}$ & $\begin{array}{l}\text { Calc. } \\
200.9\end{array}$ & $\begin{array}{c}\text { Exp. } \\
207.2\end{array}$ & $\begin{array}{l}\text { Calc. } \\
211.3\end{array}$ \\
\hline
\end{tabular}

ZB CdSe, which compares well with the experimental values measured by XRD. For the calculation of phonon frequencies, the dynamical matrix was obtained on a mesh of $k$ points using the perturbation-theory linear-response approach. Figure 1 displays the phonon dispersion along high symmetry lines for $\mathrm{W}$ and ZB CdSe. Optical modes, which mainly involve the vibration of Se atoms, span a frequency range between 170 and $206 \mathrm{~cm}^{-1}$, and a phonon gap of $45 \mathrm{~cm}^{-1}$ exists between the optical and acoustic bands owing to the atomic mass difference. The phonon density of states (PDOS) shows three distinct bands corresponding to optical, longitudinal acoustic (LA) and transverse acoustic (TA) branches which are very similar in both structures, the main difference being that the longitudinal acoustic PDOS peaks at a higher frequency in the $\mathrm{W}$ structure because of the contribution of the optical-like folded-acoustic branches.

It is well known that LDA overestimates the dielectric constant, and this affects the calculated LO-TO splitting [15]. From the DFT calculations, $\varepsilon_{\infty}$ values of 7.18 and 8.73 are obtained for the $\mathrm{W}$ and $\mathrm{ZB}$ phases, respectively. By using the corresponding experimental $\varepsilon_{\infty}$ values of 6.1 [16] and 6.56 [17], and taking into account that the squared ion plasma frequency is inversely proportional to the dielectric constant, the DFT LO frequencies can be corrected for the overestimation of $\varepsilon_{\infty}[15,18]$. A list of the corrected frequency values for the Raman active modes is given in Table 1.

3.2 Raman scattering measurements Figure 2 displays the Raman spectra of the ZB and $\mathrm{W}$ epilayers obtained in backscattering configuration from (001) and (0001) faces, respectively. In these configurations, only the LO mode is allowed in the $\mathrm{ZB}$ crystal, whereas the $E_{2}$ and $A_{1}(\mathrm{LO})$ modes are allowed in the $\mathrm{W}$ crystal. Although the $514.5 \mathrm{~nm}$ excitation is far from the $E_{0}+\Delta_{0}$ critical points [19], resonance enhancement effects are observed in the Raman spectra owing to the significant breadth of this optical transition. In the Raman spectra of ZB-CdSe, a significant intensity of the 2LO overtone, which is similar to that of the LO Raman peak, is observed, and a 3LO overtone can also be detected. A residual luminescence background picked up from the ZnTe substrate $\left(E_{g}=2.26\right.$ $\mathrm{eV})$ has been subtracted in the spectrum shown in Fig. 2.
The strong absorption through the 550-nm thick ZB-CdSe epilayer completely attenuates any Raman signal from the $\mathrm{ZnTe}$ substrate. This has been corroborated by performing Raman measurements with longer wavelength excitation lines that allow the detection of distinct Raman peaks from the ZnTe substrate.

The luminescence background is absent in the spectrum of the W CdSe epilayer. The resonant enhancement of the LO overtones up to third order is also observed in the $\mathrm{W}$ sample, although the $2 \mathrm{LO} / \mathrm{LO}$ intensity ratio is somewhat lower. The most striking difference between the Raman spectra of the W and ZB samples is the sharp, intense $E_{2}^{\text {low }}$ peak at $33 \mathrm{~cm}^{-1}$ that dominates the W CdSe spectrum. The extremely long phonon lifetimes of the lowlying $E_{2}$ optical modes is a characteristic feature of many $\mathrm{W}$ crystals arising from the lack of efficient anharmonic decay channels at such low energies $[18,20]$. Indeed, the W CdSe Raman spectrum of Fig. 2 indicates that the intrinsic $E_{2}^{\text {low }}$ line width is well below the spectral bandwidth of the spectrometer. The observation of the $E_{2}^{\text {low }}$ mode, which in the $\mathrm{W}$ structure arises from the symmetry lowering and the consequent zone-folding of the TA branch along the $\Gamma-\mathrm{A}$ direction, is a distinctive, unambiguous feature that allows one to identify the $\mathrm{W}$ phase of CdSe. As can be seen in

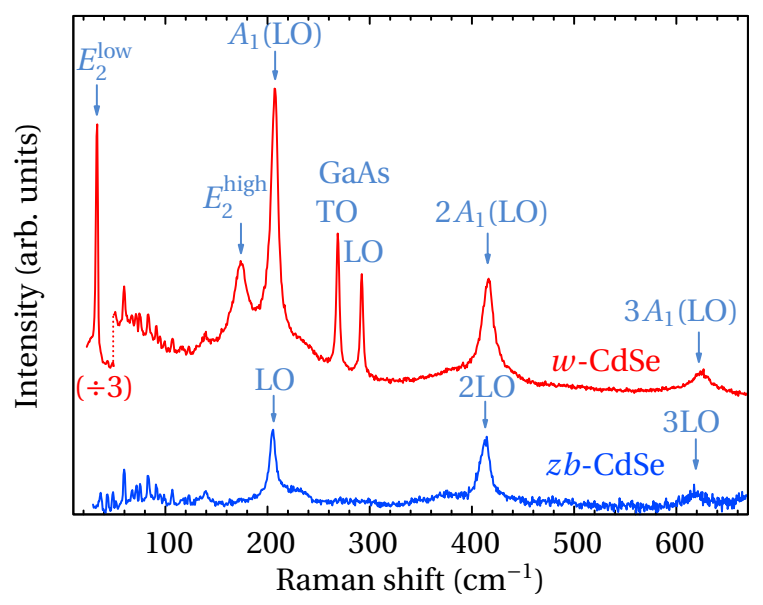

Figure 2 Room temperature Raman spectra of W and ZB CdSe epilayers. 
Fig. 2, no Raman signal from the ZB CdSe crystal is detected in this frequency range (the sharp periodic lines observed below $100 \mathrm{~cm}^{-1}$ are due to rotational modes of the air molecules). The $E_{2}^{\text {high }}$ mode at $174 \mathrm{~cm}^{-1}$ is also characteristic of the $\mathrm{W}$ phase, but this feature is less conclusive as its frequency lies close to that of the TO mode of the ZB structure (see Table 1).

Very weak broad bands are observed around 240 and $370 \mathrm{~cm}^{-1}$ in both $\mathrm{W}$ and ZB CdSe Raman spectra. The location of these bands agrees well with the calculated phonon density of states (see Fig. 1) with the wave number axis scaled by a factor of 2 , and therefore they can be assigned to second order overtones of LA and TO modes, respectively. The weak feature observed at $138 \mathrm{~cm}^{-1}$ could be due to a TO-TA combination band. The layer thickness of the W sample is low enough to allow the observation of the TO and LO modes of the GaAs substrate at 269 and $292 \mathrm{~cm}^{-1}$, respectively. It is worth noting that the Raman spectra of both samples display narrow LO peaks, with a full width at half maximum (FWHM) of 8.3 and 7.0 $\mathrm{cm}^{-1}$ for the $\mathrm{W}$ and $\mathrm{ZB}$ forms, respectively. These values compare favorably to FWHM values previously reported in nanocrystals (for instance, $\sim 12 \mathrm{~cm}^{-1}$ in [10]), which substantiates the high quality of the epitaxial single crystals used in the present study.

3.3 Strain and mode-mixing effects As can be seen in Table 1, except for the $E_{2}^{\text {low }}$ mode the experimental frequencies obtained from the room temperature Raman scattering measurements are $4-7 \mathrm{~cm}^{-1}$ lower than the calculated values. Since DFT calculations model the system implicitly at $0 \mathrm{~K}$, the calculated frequencies do not include thermal renormalization due to anharmonic interactions. These effects tend to decrease the phonon frequencies as temperature increases, and the difference between the CdSe frequencies measured at RT and the DFT calculations are consistent with the typical magnitude of the anharmonic shifts usually observed in binary semiconductors [18]. It should be noted that the $E_{2}^{\text {low }}$ modes of the W structure, related to the TA modes of the $\mathrm{ZB}$ structure, exhibit a negative Grüneisen parameter, which may compensate the anharmonic shift [18]. Although the DFT calculations yield $\mathrm{LO}$ frequencies which are nearly identical for the $\mathrm{W}$ and $\mathrm{ZB}$ structures, the LO mode frequency in the Raman spectrum of $\mathrm{ZB} \mathrm{CdSe}$ is $\sim 2 \mathrm{~cm}^{-1}$ lower than in the $\mathrm{W}$ CdSe Raman spectrum. As already discussed above, the XRD measurements reveal a residual tensile biaxial strain in the studied ZB-CdSe epilayer. To evaluate the effect of the strain on the LO phonon frequency, we have performed DFT calculations for the tetrahedral distortion $\left(a_{\|} / a_{\perp}\right.$ ratio) obtained from the XRD measurements. We obtain an in-plane strain of $\varepsilon_{\perp}=3.1 \times 10^{-3}$ and a corresponding LO phonon shift of $\Delta \omega_{\mathrm{LO}}=-1.7 \mathrm{~cm}^{-1}$ relative to the unstrained value reported in Table 1 . Therefore, the difference in the $\mathrm{LO}$ frequencies observed in the $\mathrm{W}$ and $\mathrm{ZB} \mathrm{CdSe}$ epilayers can be mainly attributed to the residual tensile strain in the ZB sample. In this regard, it is interesting to note that identical LO Raman frequencies were observed in $\mathrm{W}$ and ZB CdSe nanocrystals [10], despite the fact that surface reconstruction and lattice contraction effects were reported to take place in nanocrystalline $\mathrm{W} \mathrm{CdSe}$ [11]. It should be noted that in nanocrystals the scattering geometry is not as well defined as in the case of oriented epilayers. This may lead to mode mixing in the $\mathrm{W}$ phase, giving rise to quasi-LO modes with frequencies between the $A_{1}(\mathrm{LO})$ and $E_{1}(\mathrm{LO})$ modes [12]. Then, a lower quasiLO frequency should be expected in the Raman spectra of nanocrystalline $\mathrm{W} \mathrm{CdSe}$ that could partly compensate the lattice contraction effects and explain the accord between $\mathrm{LO}$ frequencies in $\mathrm{W}$ and $\mathrm{ZB}$ colloidal nanocrystals.

4 Conclusions We have performed a Raman scattering study of the W and ZB forms of CdSe on high quality, single-crystal epitaxial layers. The W CdSe Raman spectra exhibits a intense, characteristic $E_{2}^{\text {low }}$ peak at $33 \mathrm{~cm}^{-1}$ that allows the $\mathrm{W}$ and $\mathrm{ZB}$ forms of $\mathrm{CdSe}$ to be unambiguously identified. By contrast, the differentiation between both phases is more difficult on the basis of the LO Raman peak. Our DFT calculations indicate that the LO frequencies of the $\mathrm{W}$ and $\mathrm{ZB}$ forms are virtually identical, and the LO frequency shift observed in the ZB epilayer can be attributed to residual tensile strain. In nanocrystalline samples the frequency of this mode can be further affected by subtle strain and mode-mixing effects.

Acknowledgements This work has been supported by the Spanish MINECO under Contract No. MAT2015-71035-R.

\section{References}

[1] R. Tena-Zaera, J. Elias, and C. Lévy-Clément, Appl. Phys. Lett. 93(23), 233119 (2008).

[2] C. Y. Yeh, Z. W. Lu, S. Froyen, and A. Zunger, Phys. Rev. B 46(16), 10086-10097 (1992).

[3] N. Samarth, H. Luo, J. K. Furdyna, S. B. Qadri, Y. R. Lee, A. K. Ramdas, and N. Otsuka, Appl. Phys. Lett. 54(26), 2680 (1989).

[4] R. Tena-Zaera, A. Katty, S. Bastide, and C. Lévy-Clément, Chem. Mater. 19(7), 1626-1632 (2007).

[5] L. Manna, D. J. Milliron, A. Meisel, E. C. Scher, and A. P. Alivisatos, Nat. Mater. 2(6), 382-385 (2003).

[6] B. Sun, H. J. Snaith, A. S. Dhoot, S. Westenhoff, and N. C. Greenham, J. Appl. Phys. 97(1), 014914 (2005).

[7] C. L. Choi, H. Li, A. C. K. Olson, P. K. Jain, S. Sivasankar, and A. P. Alivisatos, Nano Lett. 11(6), 2358-2362 (2011).

[8] Y. A. Yang, H. Wu, K. R. Williams, and Y. C. Cao, Angew. Chem. Int. Ed. 44(41), 6712-6715 (2005)

[9] D. V. Talapin, J. H. Nelson, E. V. Shevchenko, S. Aloni, B. Sadtler, and A. P. Alivisatos, Nano Lett. 7(10), 29512959 (2007).

[10] A. M. Kelley, Q. Dai, Z. jie Jiang, J. A. Baker, and D. F. Kelley, Chem. Phys. 422(aug), 272-276 (2013).

[11] J. Y. Zhang, X. Y. Wang, M. Xiao, L. Qu, and X. Peng, Appl. Phys. Lett. 81(11), 2076 (2002).

[12] E. Alarcón-Llado, J. Ibáñez, R. Cuscó, L. Artús, J.D. Prades, S. Estradé, and J. R. Morante, J. Raman Spectrosc. 42(2), 153-159 (2011). 
[13] U. Rößler, E. C. F. da Silva, H. Landolt, R. Börnstein, and W. Martienssen (eds.), Numerical Data and Functional Relationships in Science and Technology: New Series. Vol. 44 (E) (Springer, Berlin, 2012).

[14] X. Gonze et al., Comput. Mater. Sci. 25, 478-492 (2002).

[15] J. Serrano, A. H. Romero, F. J. Manjón, R. Lauck, M. Cardona, and A. Rubio, Phys. Rev. B 69(9) (2004).

[16] H. W. Verleur and A. S. Barker, Phys. Rev. 155(3), 750763 (1967).

[17] C. Janowitz, O. Gnther, G. Jungk, R. L. Johnson, P. V. Santos, M. Cardona, W. Faschinger, and H. Sitter, Phys. Rev. B 50(4), 2181-2187 (1994).

[18] N. Domènech-Amador, R. Cuscó, L. Artús, T. Yamaguchi, and Y. Nanishi, Phys. Rev. B 83(24), 245203 (2011).

[19] Y. D. Kim, M. V. Klein, S. F. Ren, Y. C. Chang, H. Luo, N. Samarth, and J. K. Furdyna, Phys. Rev. B 49(11), 7262 7270 (1994).

[20] R. Cuscó, E. Alarcón-Lladó, J. Ibáñez, L. Artús, J. Jiménez, B. Wang, and M. J. Callahan, Phys. Rev. B 75(16), 165202 (2007). 